\section{Multidimensional Filters for High-Speed Processing}

\section{K. M. TY AND A. N. VENETSANOPOULOS}

Abstract-A new digital filter structure is developed for the realization of multidimensional recursive filters used in high-speed processing. The structure possesses a high throughput rate, which depends on the dimensionality $m$ of the filter, but is independent of its order. The features that contribute to its high-speed performance include both parallelism and pipelining. The clock cycle of the proposed filter structure is derived and expressed in terms of the time required for each arithmetic operation.

\section{INTRODUCTION}

Multidimensional ( $m$-D) signal processing requires an enormous number of arithmetic operations to be performed per second. One of the feasible solutions to this demanding task is to use multiprocessors [1]. However, with a suitable separation of the processing into stages, a fully pipelined parallel-processing architecture can be developed. One-dimensional (1-D) and two-dimensional (2-D) high-speed digital filter structures have been reported in [2]-[5]. In this correspondence, a fast filter structure for the implementation of a general $m$-D recursive digital filter is proposed. Due to the speed limitation of data propagation in the feedback loop [2], recursive filters are being considered. The motivation behind this contribution is to provide insight into the dependency of the filter throughput rate on its dimensionality and order.

\section{A High-Speed Structure for $m$-D Recursive Filters}

An $m$-D casual recursive digital filter is described by the linear difference equation

$$
\begin{aligned}
y_{n_{1}, n_{2}, \cdots, n_{m}}= & \sum_{i_{1}=0}^{M_{1}} \sum_{i_{2}=0}^{M_{2}} \cdots \sum_{i_{m}=0}^{M_{m}} a_{i_{1}, i_{2}, \cdots, i_{m}} x_{n_{1}, n_{2}, \cdots, n_{i m}} \\
& -\sum_{\substack{k_{1}=0 \\
k_{1}+}}^{N_{1}} \sum_{\substack{k_{2}=0 \\
k_{2}+}}^{N_{2}} \cdots \sum_{\substack{k_{m}=0 \\
k_{m} \neq 0}}^{N_{m}} b_{k_{1}, k_{2}, \cdots, k_{m}} y_{n_{1}-k_{1}, n_{2}-k_{2}, \cdots, n_{m}-k_{m}}
\end{aligned}
$$

where the $x$ 's and $y$ 's are the input and the output $m$-D data, respectively, and $a_{i_{1}, i_{2}, \cdots, i_{m}}$ and $b_{k_{1}, k_{2}, \cdots, k_{m}}$ are the filter coefficients of the nonrecursive and recursive blocks, respectively. The digital filter described by (1) has the transfer function

$$
\begin{aligned}
& H\left(z_{1}^{-1}, z_{2}^{-1}, \cdots, z_{m}^{-1}\right)
\end{aligned}
$$

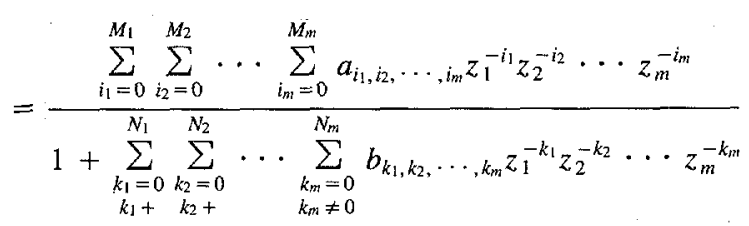

where $z_{i}^{-1}$ 's $, i=1,2, \cdots, m$ are the unit delays along different orthogonal axes of the $m$-D space. To design a high-speed structure for the filter described by (2), we have to find out the shortest possible critical path length as in [3] and [4]. The critical path is the longest path among all the possible paths from the output of a delay element to the input of the same or another one.

The transposition of the direct form network [6], as shown in Fig. 1, is the important key to this high-speed filter structure. This

Manuscript received May 3, 1986; revised October 29, 1986.

The authors are with the Department of Electrical Engineering, University of Toronto, Toronto, Ont., Canada M5S 1A4.

IEEE Log Number 8613875

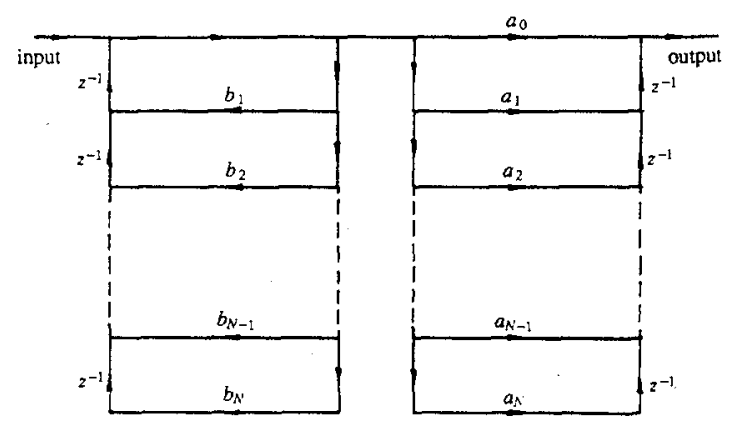

Fig. 1. Transposition of the direct form network of a 1-D filter.

is due to the fact that after transposition, the new filter network possesses parallelism, i.e., multipliers operating in parallel, as well as pipelining, i.e., partial sums of products are stored in the delay elements. Mathematically, the transposition can be expressed using Horner's rule [7]. The numerator of the filter transfer function $N\left(z_{1}^{-1}, z_{2}^{-1}, \cdots, z_{m}^{-1}\right)$ can equivalently be rewritten as

$$
\begin{aligned}
N\left(z_{1}^{-1}, z_{2}^{-1}, \cdots, z_{m}^{-1}\right)= & a_{0}, \cdots, 0+z_{I}^{-1} P_{1}\left(z_{1}^{-1}\right) \\
& +z_{2}^{-1} P_{2}\left(z_{1}^{-1}, z_{2}^{-1}\right)+\cdots \\
& +z_{m}^{-1} P_{m}\left(z_{1}^{-1}, z_{2}^{-1}, \cdots, z_{m}^{-1}\right)
\end{aligned}
$$

where

$$
\begin{aligned}
& P_{1}\left(z_{1}^{-1}\right)=a_{1,0}, \cdots, 0+z_{1}^{-1}\left(a_{2,0}, \cdots, 0+z_{1}^{-1}\right. \\
& \cdot\left(a_{3,0}, \cdots, 0+\cdots \cdot\right. \\
& \left.\left.+z_{1}^{-1}\left(a_{M_{1}, 0}, \cdots, 0\right) \cdots\right)\right) \\
& P_{2}\left(z_{1}^{-1}, z_{2}^{-1}\right)=a_{0,1,0} \cdots, 0+z_{1}^{-1}\left(a_{1,1,0}, \cdots, 0\right. \\
& +z_{1}^{-1}\left(a_{2,1,0, \cdots, 0}+z_{1}^{-1}\left(a_{3,1,0}, \cdots, 0\right.\right. \\
& \left.\left.+\cdots+z_{1}^{-1}\left(a_{M_{1}, 1,0}, \cdots, 0\right) \cdots\right)\right) \\
& +z_{2}^{-1}\left[a_{0,2,0} \cdots, 0+z_{1}^{-1}\left(a_{1,2,0, \cdots, 0}\right.\right. \\
& +z_{1}^{-1}\left(a_{2,2,0, \cdots, 0}+z_{1}^{-1}\left(a_{3,2,0}, \cdots, 0\right.\right. \\
& \left.\left.\left.+\cdots+z_{1}^{-1}\left(a_{M_{1}, 2,0}, \cdots, 0\right) \cdots\right)\right)\right) \\
& +\cdots+z_{2}^{-1}\left[a_{0, M_{2}, 0, \cdots, 0}\right. \\
& +z_{1}^{-1}\left(a_{1, M_{2}, 0}, \cdots, 0+z_{1}^{-1}\left(a_{2, M_{2}, 0, \cdots, 0}\right.\right. \\
& +\cdots+z_{1}^{-1}\left(a_{M_{1}, M_{2}, 0}, \cdots, 0\right) \\
& \cdots)] \cdot \cdots \\
& P_{m}\left(z_{1}^{-1}, z_{2}^{-1}, \cdots, z_{m}^{-1}\right) \\
& =a_{0, \cdots, 0, M_{m}}+z_{1}^{-1}(\cdots)+z_{2}^{-1}(\cdots)+\cdots+z_{m}^{-1}(\cdots \\
& \left.+z_{m}^{-1}(\cdots) \cdot \cdots\right) \text {. }
\end{aligned}
$$

If we consider $N\left(z_{1}^{-1}, z_{2}^{-1}, \cdots, z_{m}^{-1}\right)$ as an FIR filter, its critical path length is shown in Fig. 2.

Similarly, the denominator of the filter transfer function $D\left(z_{1}^{-1}\right.$, $\left.z_{2}^{-1}, \cdots, z_{m}^{-1}\right)$ can also be expressed as the form in (3), with $a_{0, \ldots, 0}$ replaced by 1 and all $a$ 's replaced by $b$ 's with the subscripts $M$ changed to $N$. Thus, 


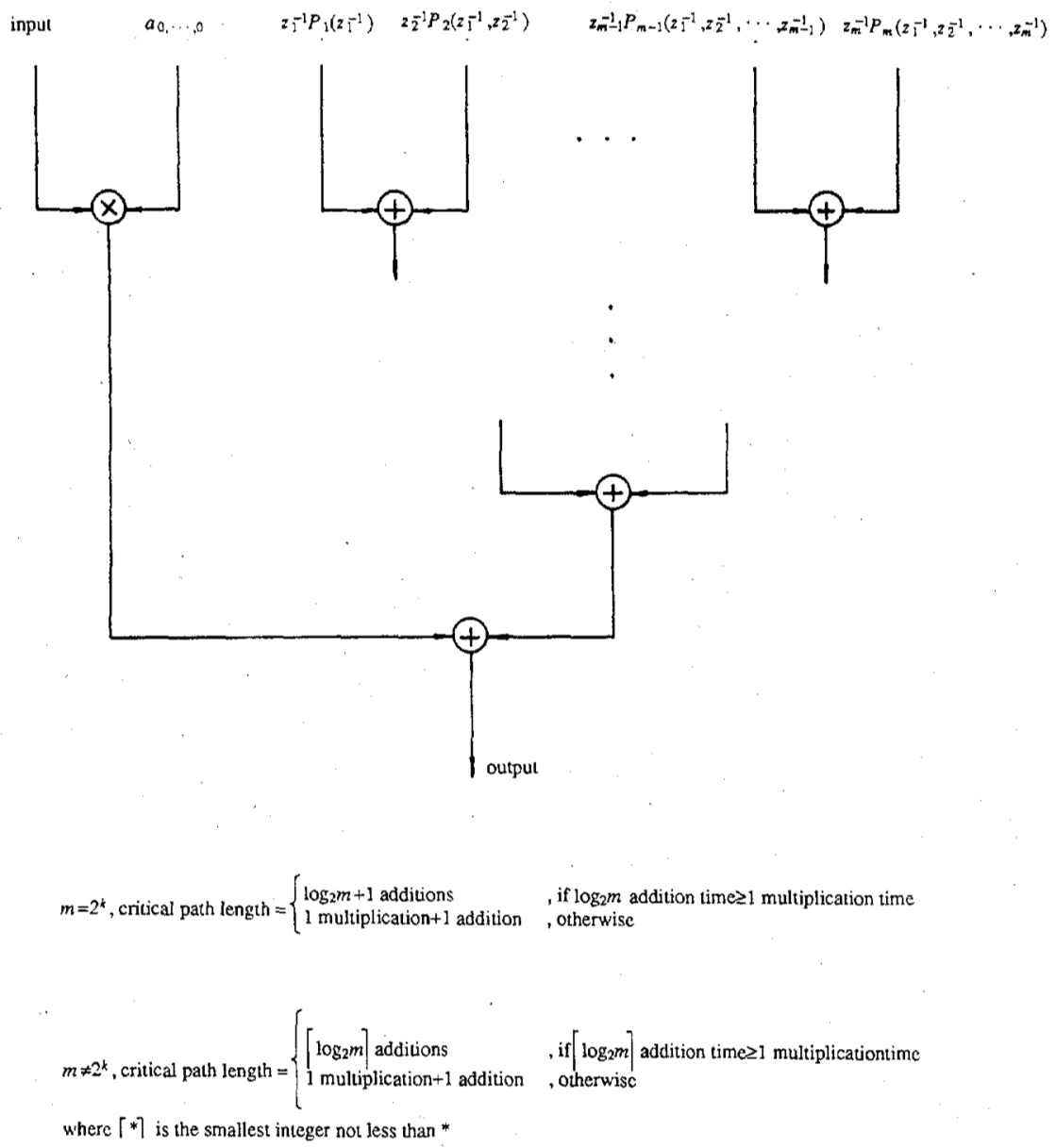

Fig. 2. Critical path length of nonrecursive filter.

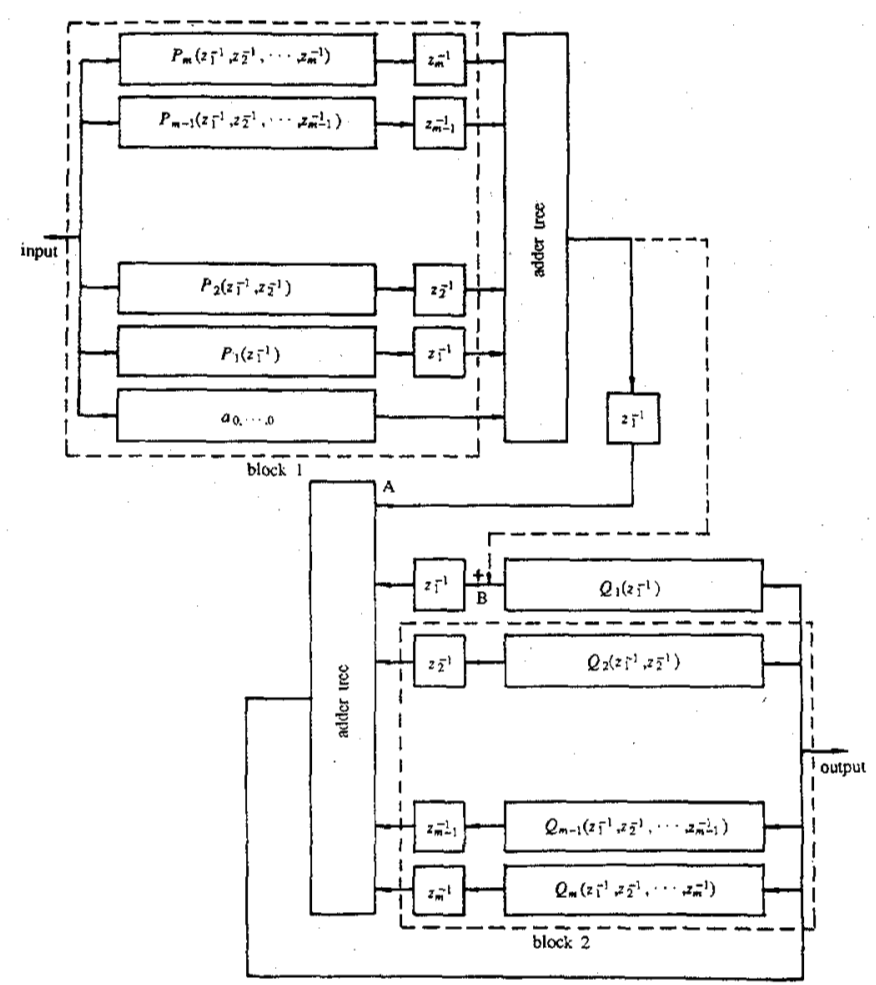

Fig. 3. Cascade of nonrecursive and recursive blocks.

$$
\begin{aligned}
D\left(z_{1}^{-1}, z_{2}^{-1}, \cdots,\right. & \left.z_{m}^{-1}\right) \\
= & 1+z_{1}^{-1} Q_{1}\left(z_{1}^{-1}\right)+z_{2}^{-1} Q_{2}\left(z_{1}^{-1}, z_{2}^{-1}\right) \\
& +\cdots+z_{m}^{-1} Q_{m}\left(z_{1}^{-1}, z_{2}^{-1}, \cdots, z_{m}^{-1}\right) .
\end{aligned}
$$

The cascade of $N\left(z_{1}^{-1}, z_{2}^{-1}, \cdots, z_{m}^{-1}\right)$ and $1 / D\left(z_{1}^{-1}\right.$ $\left.z_{2}^{-1}, \cdots, z_{m}^{-1}\right)$ will give the original filter transfer function $H\left(z_{1}^{-1}, z_{2}^{-1}, \cdots, z_{m}^{-1}\right)$. Yet, even with the transposed structure, the resulting filter structure does not have the shortest possible critical path [2]. This is due to the fact that every input has to be processed by the nonrecursive and recursive block of the filter; thus, the whole filtering process could be divided into two stages.

To achieve a minimum cycle structure, the original filter transfer function is modified as follows:

$$
\tilde{H}\left(z_{1}^{-1}, z_{2}^{-1}, \cdots, z_{m}^{-1}\right)=H\left(z_{1}^{-1}, z_{2}^{-1}, \cdots, z_{m}^{-1}\right) z_{1}^{-1} \text {. }
$$

The $z_{1}^{-1}$ delay is arbitrarily chosen. The new output sequence $\tilde{y}_{n_{1}, n_{2}, \cdots n_{m}}=y_{n_{1}-1, n_{2}, \cdots n_{n+1}}$. To realize the modified filter transfer function, the simplest way is to insert a unit delay $z_{1}^{-1}$ in the path connecting the nonrecursive and recursive blocks of the filter as shown in Fig. 3, with output of the nonrecursive block connected to point $\boldsymbol{A}$. However, this is not desirable because the adder tree will have $m+1$ inputs, possibly resulting in an increase of the critical path length. If the connection is made at point $B$, the number of inputs to the adder tree of the recursive block remains $m$, and the extra $z_{1}^{-1}$ is also eliminated. The two remaining problems are the detailed connection at point $B$ and the critical path length. The first one is shown in Fig. 4. The output of the $z_{1}^{-1}$ is input to the adder tree of the nonrecursive block since this configuration can possibly reduce the critical path length, i.e., $\left\lceil\log _{2}(m+2)\right\rceil \leq$ 


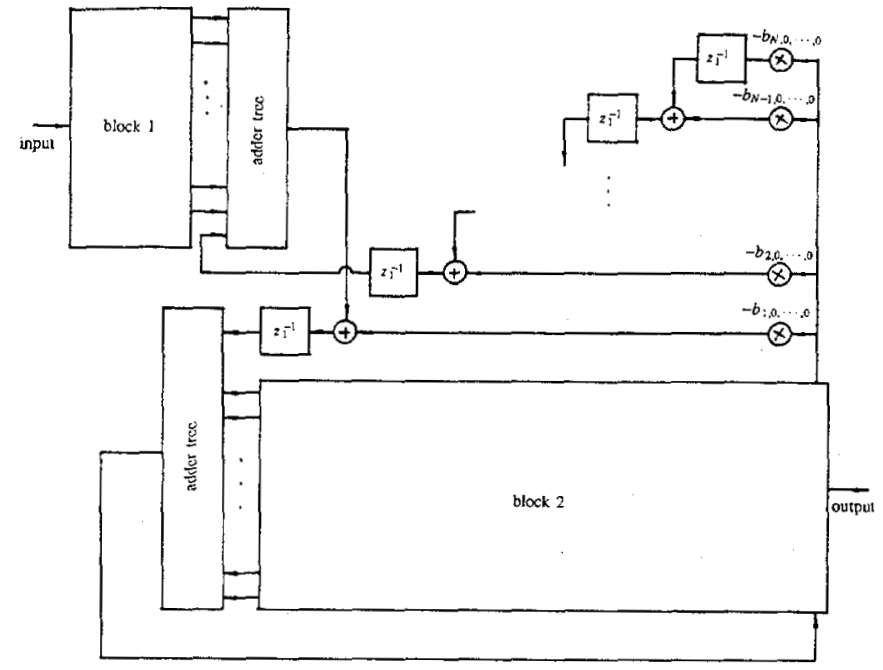

Fig. 4. Modified parallel-pipelined filter structure.

$1+\left\lceil\log _{2}(m+1)\right\rceil$. From the same figure, the critical path length $\left(c_{r}\right)$ is

$$
c_{r}=\left\{\left\lceil\log _{2} m\right\rceil+1\right\} \text { addition(s) }+1 \text { multiplication. }
$$

The critical path length given by (7) can be considered to be the worst case. Moreover, the critical path length is only a function of the dimensionality of the filter, not its order. Equation (7) has already been reported in the literature for the special cases of 1-D [2] and 2-D filters [3], [4].

\section{EXAMPLE} $[8]$

Consider the 3-D IIR fan filter described by the transfer function

$$
H\left(z_{1}^{-1}, z_{2}^{-1}, z_{3}^{-1}\right)=\frac{N\left(z_{1}^{-1}, z_{2}^{-1}, z_{3}^{-1}\right)}{D\left(z_{1}^{-1}, z_{2}^{-1}, z_{3}^{-1}\right)}
$$

where

$$
\begin{aligned}
N\left(z_{1}^{-1}, z_{2}^{-1}, z_{3}^{-1}\right)= & z_{1}^{-1} z_{2}^{-1}+p_{1} z_{2}^{-1}\left(z_{1}^{-2}+1\right)+p_{1} z_{1}^{-1}\left(z_{2}^{-2}\right. \\
& +1)+p_{2}\left(z_{1}^{-2}+1\right)\left(z_{2}^{-2}+1\right) \\
& +z_{3}^{-1}\left[p_{3} z_{1}^{-1} z_{2}^{-1}+p_{4} z_{2}^{-1}\left(z_{1}^{-2}+1\right)\right. \\
& +p_{4} z_{1}^{-1}\left(z_{2}^{-2}+1\right) \\
& \left.+p_{5}\left(z_{1}^{-2}+1\right)\left(z_{2}^{-2}+1\right)\right] \\
D\left(z_{1}^{-1}, z_{2}^{-1}, z_{3}^{-1}\right)= & {\left[1+p_{6}\left(z_{1}^{-2}+1\right) z_{2}^{-1} z_{3}^{-1}\right] } \\
& {\left[1+p_{6}\left(z_{2}^{-2}+1\right) z_{1}^{-1} z_{3}^{-1}\right] }
\end{aligned}
$$

The filter coefficients are as follows:

$$
\begin{aligned}
& p_{1}=0.8917186 \\
& p_{2}=-0.1226214 \times 10 \\
& p_{3}=0.5509735 \times 10^{2} \\
& p_{4}=0.1464239 \times 10^{2} \\
& p_{5}=0.1274755 \times 10 \\
& p_{6}=0.2900793 \times 10^{-1} .
\end{aligned}
$$

According to (3) and (4), (9) can be rewritten as

$$
\begin{aligned}
N\left(z_{1}^{-1}, z_{2}^{-1}, z_{3}^{-1}\right)= & p_{2}+z_{1}^{-1} P_{1}\left(z_{1}^{-1}\right)+z_{2}^{-1} P_{2}\left(z_{1}^{-1}, z_{2}^{-1}\right) \\
& +z_{3}^{-1} P_{3}\left(z_{1}^{-1}, z_{2}^{-1}, z_{3}^{-1}\right)
\end{aligned}
$$

with

$$
P_{1}\left(z_{1}^{-1}\right)=p_{1}+p_{2} z_{1}^{-1}
$$

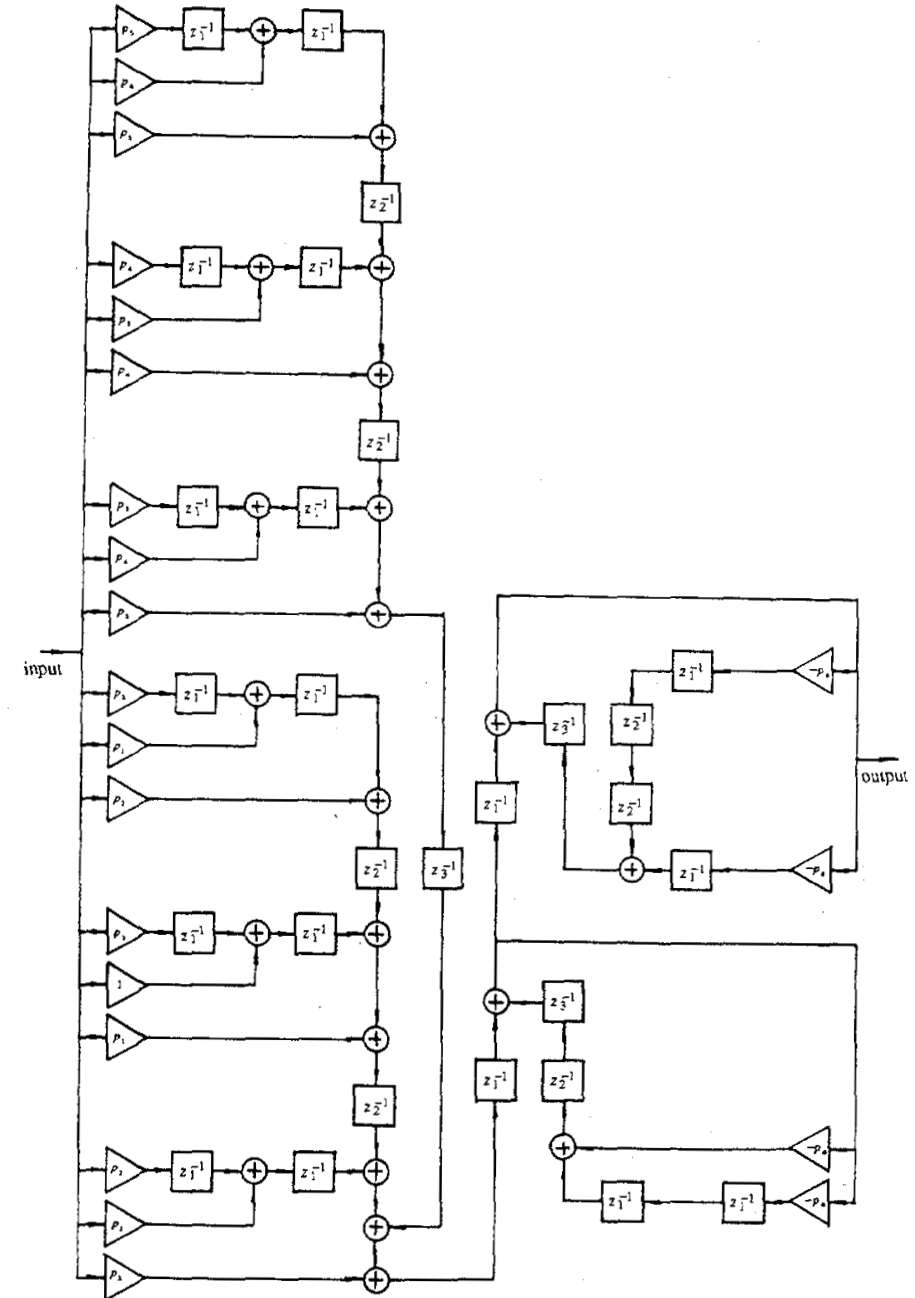

Fig. 5. Proposed high-speed structure for a 3-D IIR fan filter.

$$
\begin{aligned}
P_{2}\left(z_{1}^{-1}, z_{2}^{-1}\right)= & p_{1}+z_{1}^{-1}\left(1+p_{1} z_{1}^{-1}\right) \\
& +z_{2}^{-1}\left[p_{2}+z_{1}^{-1}\left(p_{1}+p_{2} z_{1}^{-1}\right)\right] \\
P_{3}\left(z_{1}^{-1}, z_{2}^{-1}, z_{3}^{-1}\right)= & p_{5}+z_{1}^{-1}\left(p_{4}+p_{5} z_{1}^{-1}\right) \\
& +z_{2}^{-1}\left\{p_{4}+z_{1}^{-1}\left(p_{3}+p_{4} z_{1}^{-1}\right)\right. \\
& \left.+z_{2}^{-1}\left[p_{5}+z_{1}^{-1}\left(p_{4}+p_{5} z_{1}^{-1}\right)\right]\right\} .
\end{aligned}
$$

Similarly, (10) can also be rewritten as

$$
\begin{aligned}
D\left(z_{1}^{-1}, z_{2}^{-1}, z_{3}^{-1}\right)= & {\left[1+z_{1}^{-1} Q_{1}^{1}\left(z_{1}^{-1}\right)+z_{2}^{-1} Q_{2}^{1}\left(z_{1}^{-1}, z_{2}^{-1}\right)\right.} \\
& \left.+z_{3}^{-1} Q_{3}^{1}\left(z_{1}^{-1}, z_{2}^{-1}, z_{3}^{-1}\right)\right] \\
& \times\left[1+z_{1}^{-1} Q_{1}^{2}\left(z_{1}^{-1}\right)+z_{2}^{-1} Q_{2}^{2}\left(z_{1}^{-1}, z_{2}^{-1}\right)\right. \\
& \left.+z_{3}^{-1} Q_{3}^{2}\left(z_{1}^{-1}, z_{2}^{-1}, z_{3}^{-1}\right)\right]
\end{aligned}
$$

with

$$
\begin{aligned}
Q_{1}^{1}\left(z_{1}^{-1}\right) & =0 \\
Q_{2}^{1}\left(z_{1}^{-1}, z_{2}^{-1}\right) & =0 \\
Q_{3}^{1}\left(z_{1}^{-1}, z_{2}^{-1}, z_{3}^{-1}\right) & =z_{2}^{-1}\left(p_{6}+p_{6} z_{1}^{-2}\right) \\
Q_{1}^{2}\left(z_{1}^{-1}\right) & =0 \\
Q_{2}^{2}\left(z_{1}^{-1}, z_{2}^{-1}\right) & =0 \\
Q_{3}^{2}\left(z_{1}^{-1}, z_{2}^{-1}, z_{3}^{-1}\right) & =p_{6} z_{1}^{-1}+z_{2}^{-2}\left(p_{6} z_{1}^{-1}\right) .
\end{aligned}
$$

Fig. 5 shows the high-speed structure of the modified transfer function given in (8), (9), and (10). The structure shown has taken 
advantage of the factorization of the denominator. Since each recursive block contributes a delay of $z_{1}^{-1}$ to the modified transfer function,

$$
\tilde{H}\left(z_{1}^{-1}, z_{2}^{-1}, z_{3}^{-1}\right)=H\left(z_{1}^{-1}, z_{2}^{-1}, z_{3}^{-1}\right) z_{1}^{-2} .
$$

The critical path consists of one multiplication and two additions, instead of three as given by (7). The decrease in the number of additions is due to the fact that $Q_{1}^{1}\left(z_{1}^{-1}\right), Q_{2}^{1}\left(z_{1}^{-1}, z_{2}^{-1}\right), Q_{1}^{2}\left(z_{1}^{-1}\right)$, and $Q_{2}^{2}\left(z_{1}^{-1}, z_{2}^{-1}\right)$ are zeros. From the figure, it is apparent that for this specific filter, the proposed structure requires only six coefficients for the implementation, taking advantage of the symmetrical properties of the filter.

\section{CONClusions}

A high-speed filter structure for the implementation of $m$-D filters with great modularity, parallelism, and pipelining was proposed. The throughput rate depends only on its dimensionality $m$, and does not depend on its order. An example was given to illustrate the effect of cascading more than two blocks. One of the applications of multidimensional filtering would be in the area of image sequence filtering where temporal filtering would also be involved. This application requires real-time filtering. With the advances in VLSI techology and digital signal processing chips, the proposed structure can be used for the implementation of the 3-D filters required in this application.

\section{REFERENCES}

[1] A. N. Venetsanopoulos and V. Cappellini, "Real-time image processing," in Multidimensional Systems: Techniques and Applications, S. G. Tzafestas, Ed. New York: Marcel Dekker, 1986, ch. 8, pp. 345399.

[2] D. Dubois and W. Steenaart, "High speed stored product recursive digital filters," IEEE Trans. Circuits Syst., vol. CAS-29, pp. 390393 , June 1982.

[3] K. M. Ty and A. N. Venetsanopoulos, "Two-dimensional digital filters with minimum cycle time," in Proc. ICASSP, vol. 4, Tampa, FL, Mar. 1985, pp. 1527-1530.

[4] —_, "A fast filter for real-time image processing," IEEE Trans. Circuits Syst., vol. CAS-33, pp. 948-957, Oct. 1986.

[5] A. N. Venetsanopoulos, K. M. Ty, and A. C. P. Loui, "Real-time image processing," in V Polish-English Seminar Real-Time Process Contr., Warzawa, Poland, 1986.

[6] A. V. Oppenheim and R. W. Schafer, Digital Signal Processing. En glewood Cliffs, NJ: Prentice-Hall, 1975.

[7] V. A. Dyck, J. D. Lawson, and J. A. Smith, Introduction to Computing. Reston, VA: Reston, 1979.

[8] A. N. Venetsanopoulos and C. L. Nikias, "Design and realization of multidimensional digital filters via matrix decomposition approaches," Adv. Geophys. Data Processing, vol. 2, pp. 263-305, 1985.

\section{An Eigenvalue Recursion for Toeplitz Matrices}

\author{
D. M. WILKES AND M. H. HAYES
}

\footnotetext{
Abstract-In this correspondence, a recursive algorithm for finding eigenvalues of a real symmetric Toeplitz matrix from the eigenvalues of nested Toeplitz submatrices is presented. Given the eigenvalues of two nested Toeplitz submatrices, the eigenvalues of the next larger

Manuscript received June 24, 1986; revised January 12, 1987. This work was supported in part by the National Science Foundation under Grant ECS8352161 , the GTE Laboratories, and by the Joint Services Electronics Program under Contract DAAG29-84-K-0024.

The authors are with the School of Electrical Engineering, Georgia Institute of Technology, Atlanta, GA 30332.

IEEE Log Number 8714195.
}

Toeplitz matrix can be found simply by solving a Vandermonde set of equations and rooting two polynomials.

\section{INTRODUCTION}

Toeplitz matrices occur in a wide variety of applications. For example, in communication theory, Toeplitz matrices appear as the covariance matrices of stationary random processes. In digital signal processing, they are commonly found in speech analysis, linear prediction, and power spectrum estimation. Recently, there has been a great deal of interest in the eigenvalues and eigenvectors of Toeplitz matrices since they appear in solutions to the harmonic retrieval problem [1], [2] and have been used to improve linear prediction and power spectrum estimates [3]. Previous work concerned with the eigenvalues of Toeplitz matrices has often emphasized the distribution of eigenvalues as the size of the matrix tends to infinity [4], [5]. However, there is a growing body of literature on the spectral properties of finite-dimensional Toeplitz matrices [6]-[10]. In particular, Delsarte and Genin [6] have produced an important work containing most of the presently known properties of these matrices. In the body of this correspondence is an equation which shows that, at least in theory, the eigenvalues of symmetric Toeplitz matrices may be computed recursively. However, this equation contains a term of the form adj $[\lambda I-A]$ where $A$ is a symmetric matrix and $\lambda$ is a parameter to be varied. This adjoint computation is equivalent to finding a matrix inverse symbolically, and thus is not suitable for practical calculations. In this correspondence, a different but computable recursive algorithm for finding the eigenvalues of a real symmetric Toeplitz matrix from the eigenvalues of nested Toeplitz submatrices is presented. Given the eigenvalues of two nested Toeplitz submatrices, the eigenvalues of the next larger Toeplitz matrix can be found simply by solving a Vandermonde set of equations and rooting two polynomials.

\section{A New Eigenvalue Recursion for Symmetric Toeplitz MATRICES}

An $n \times n$ symmetric Toeplitz matrix has the form

$$
C_{n}=\left[\begin{array}{cccc}
c_{0} & c_{1} & \cdots & c_{n-1} \\
c_{1} & c_{0} & \cdots & c_{n-2} \\
\vdots & \vdots & & \vdots \\
c_{n-1} & c_{n-2} & \cdots & c_{0}
\end{array}\right]
$$

The characteristic equation of the matrix is denoted by

$$
f_{n}(\lambda)=\operatorname{det}\left(\lambda I-C_{n}\right)=\left(\lambda-\lambda_{1}\right)\left(\lambda-\lambda_{2}\right) \cdots\left(\lambda-\lambda_{n}\right)
$$

where the $\lambda_{i}$ 's are the eigenvalues of $C_{n}$. In linear prediction, one needs to solve a set of linear equations of the form

$$
C_{n} a=E_{n-1} e_{1}
$$

where $C_{n}$ is a symmetric Toeplitz matrix, $e_{1}$ is the unit vector with a 1 in the first position and zeros everywhere else, and $E_{n-1}$ is the prediction error associated with $a$. The Levinson/Durbin recursion, which is used to solve these equations, produces the vector

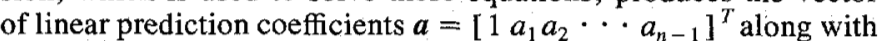
a set of reflection coefficients $k_{i}, i=1,2, \cdots, n-1$. Thus, with any $n \times n$ symmetric Toeplitz matrix, there is an associated set of linear prediction coefficients and a set of reflection coefficients.

Given an $n \times n$ Toeplitz matrix $C_{n}$, consider the family of Toeplitz matrices defined by $D(\lambda)=\left(C_{n}-\lambda I\right)$. For each value of $\lambda, D(\lambda)$ has a set of $n-1$ reflection coefficients. The reflection coefficients of $D(\lambda)$ may therefore be considered to be a set of $n-1$ functions of $\lambda$. It is important to note that $\lambda$ is used as a continuous real variable and is not necessarily an eigenvalue of $C_{n}$. If we plot $k_{n-1}$, the last reflection coefficient of $D(\lambda)$, as a function of $\lambda$, we get a plot of the form shown in Fig. 1. In this example, $n=5$ so $C_{n}$ is a $5 \times 5$ matrix and we are plotting $k_{4}$ versus $\lambda$. 